\title{
Longitudinal fluctuations in PD1 and PD-L1 expression in association with changes in anti-viral immune response in chronic hepatitis $B$
}

\author{
Zhang Wenjin ${ }^{1}$, Peng Chuanhui ${ }^{1}$, Wan Yunle ${ }^{2}$, Shaikh Abdul Lateef ${ }^{1}$ and Zheng Shusen ${ }^{1 *}$
}

\begin{abstract}
Background: Controversy exists regarding the role of PD1 and its ligand PD-L1 in chronic hepatitis B infection. In some studies, persistent HBV infection has been attributed to high levels of PD-1 and PD-L1 expression on HBV-specific T-cells and antigen-presenting cells (APCs) respectively. Other studies revealed that the up-regulation of PD-1 and PD-L1 during an acute inflammation phase is required to offset increasing positive co-stimulatory signals to avoid severe damage by an over-vigorous immune response.
\end{abstract}

Methods: Fifteen chronic hepatitis B patients, with inflammatory flare episode, were recruited prospectively. Based on serum HBV-DNA, HBsAg load, and ALT values, inflammatory flare episode were divided into initial, climax, decline and regression phase. Blood sample and liver biopsy tissues from each individual were taken in these 4 phases respectively. Circulating and intra-hepatic PD1 and PD-L1 expression levels were monitored throughout the inflammatory flare episode by flow cytometry and immunostaining and these expression levels were related to the HBV-specific T-cell changes, expression of pro-inflammatory cytokines, HBV-DNA replication and HBV antigen load.

Results: ]The levels of PD-1 and PD-L1 expressions were significantly up-regulated in the inflammation ascending phase, initial and climax period and in parallel with HBV-specific colon expansion. It showed increasing the level of serum ALT and decreasing the HBV-DNA loads. As the level of inflammation reduced, the circulating and intra-hepatic PD1 and circulating PD-L1 decreased progressively in concordance with serum ALT, HBV-DNA and HBsAg loads decreased except intra-hepatic PD-1 expression. Intra-hepatic PD-L1 expression did not decrease significantly during the regression phase of inflammation compared to that in prior period. The intra-hepatic PD-L1 expression remained relatively on higher level when serum HBV-DNA load and ALT decreased to approximately normal range.

Conclusion: The relatively high level of intra-hepatic PD-L1 expression during the inflammatory regression period may contribute to constitute an immunosuppressive microenvironment, which facilitate persistent HBV infection via the inhibition of HBV-specific T cell clonal expansion.

Keywords: PD1, PD-L1, Hepatitis B

\section{Background}

Hepatitis B virus (HBV) infection results in the heterogeneous diseases states that vary greatly in HBV replication, liver pathology damage and outcome from person to person. Some patients control infection efficiently and are able to eliminate the virus either without developing evident liver inflammation or a self-limited

\footnotetext{
* Correspondence: shusenzheng@zju.edu.cn

'Key Laboratory of Combined Multi-organ Transplantation, Ministry of Public Health, First Affiliated Hospital, School of Medicine, Zhejiang University, 79 Qingchun Road, Hangzhou 310003, China

Full list of author information is available at the end of the article
}

acute hepatitis syndrome that resolves without longterm periodic inflammatory episodes. Other patients are unable to clear the virus and develop persistent HBV infection.

Anecdotal evidence from clinical observation has shown that the chronic hepatitis B infection often manifests clinically with repeated inflammatory episodes marked by fluctuating levels of serum ALT and HBVDNA. Levels of serum HBV-DNA and HBV antigens load progressively decrease throughout the inflammatory flare. During immune inactive period, HBV antigens 
begin to re-accumulated until they reach a high enough level to trigger another round of inflammation.

In recent studies, the inability to eliminate virus in patients with chronic hepatitis $B$ has been attributed to high levels of expression of programmed death 1 (PD-1) and its ligand (PD-L1/B7-H1) on viral antigen-specific $\mathrm{T}$-cells and antigen -presenting cells (APCs) respectively [1-9]. The interaction between this pair of co-inhibitory molecules has been shown to contribute directly to T-cell dysfunction and poor control of viral replication. Blocking the PD-1/PD-L1 interaction in vitro reversed exhausted cytokine production and proliferation of these HBV-specific T cells [1,4-6]. Thus, up-regulation of PD1 and PD-L1 are generally considered markers of malfunction of the HBV-specific immune response.

In contrast, other studies revealed that negative costimulatory molecules play a key role in maintenance of homeostasis [10,11]. Studies showed that delayed up regulation of PD1 and PD-L1 were attributed to fulminant liver inflammation in patients with acute hepatitis $B$ and finally led to acute liver failure [12-14]. Previous studies revealed that in patients with acute hepatitis B, PD-L1 expression levels were up regulated during the ascending phase of inflammation and decreased when inflammation regressed. This phenomenon suggested that a balance exists between the negative and positive costimulatory molecules on activated immune cells.

In order to elucidate the role of PD1 and PD-L1 in the hepatitis $\mathrm{B}$ immune response, the relationship between the PD1/ PD-L1 expression and immune response events, such as HBV-specific CD8 T-cell expansion and contraction, pro-inflammatory cytokines expression, viral load, and disease pathogenesis should be analyzed.

The majority of previous studies of the PD1 and PD-L1 expression in chronic hepatitis B patients were retrospective and cross-sectional. In this study, patients were enrolled prospectively and inflammatory flare period were divided into initial, climax, decline and regression phase. This study is novelty in its prospective and longitudinal analysis of PD1 and PD-L1 expression in chronic hepatitis $B$ patients throughout inflammatory flare period.

The results showed that PD-1/PD-L1 expression was significantly up-regulated during the inflammatory climax period. During the regression period, when serum ALT decrease to approximately normal levels, intra-hepatic PD-L1 expression on Kupffer cells did not decrease significantly compared to that in prior phase. These data suggest that the relatively high level of intra-hepatic PD-L1 expression during the inflammatory regression period may contribute to constitute an immunosuppressive microenvironment. The immunosuppressive microenvironment in liver may facilitate persistent $\mathrm{HBV}$ infection via the transmission of co-inhibitory signals to activated T-cells, and finally lead to the next round of liver inflammation.

\section{Methods}

\section{Subjects}

Chronic hepatitis B patients were followed-up in the outpatient clinic by detection of serum ALT, HBV-DNA and HBs-Ag load. Subjects who developed hepatitis B flare episode during follow up period were enrolled in this study. Serum HBV-DNA load more than $5 \times 10^{3}$ copies $/ \mathrm{mL}$, and serum ALT level near to 5 times the upper limit of normal, were considered as the onset of initiation of inflammation. HBV tolerance patients and fulminant hepatitis B patients were excluded from this study. The recruiting work was stopped when the fifteenth patient was enrolled. Each of these fifteen patients was followed with protocol visit for a median period of 4 months (range, 3-5 months). Liver biopsy was performed according to ultrasound guide in appointed time point as mentioned following:

Written informed consent was obtained from each enrolled patient, and the study protocol was approved by the Ethics Committee of our unit. Reports of all patients were negative for anti-hepatitis $\mathrm{D}$ virus antibodies, antihepatitis C virus (anti-HCV) antibodies, anti-HIV1,2 antibodies, and auto-antibodies. The clinical data of these enrolled patients at the first time point (initial phase of inflammation) are summarized in Table 1.

Heparinized blood samples used for the separation of peripheral blood mononuclear cells (PBMCs) and liver biopsy tissue specimens were obtained from patients at 4 time points:

Time 1 (T1) - Initial inflammation phase: High HBVDNA load, a high HBsAg load, and serum ALT values were no more than 5 times the upper limit of normal (normal range: $10-40 \mathrm{IU} / \mathrm{L}$ ).

Time 2 (T2) - Climax phase of inflammation: Reduced viremia and ALT levels elevated to greater than 10 times the upper limit of normal (normal range: $10-40 \mathrm{IU} / \mathrm{L})$.

Time 3 (T3) - Decline phase of inflammation: Serum ALT levels decreased by greater than $50 \%$ of values in the $\mathrm{T} 2$ phase.

Time 4 (T4) - Regression phase of inflammation: Serum HBV-DNA decreased to the level less than $5 \times 10^{3}$ copies/mL and serum ALT level less than $100 \mathrm{IU} / \mathrm{L}$.

\section{Hepatitis serology}

HBsAg, anti-HBs, anti-HBc, HBeAg,anti-HBe, anti-HDV, anti-HCV, and anti-HIV titers were measured using an enzyme immunoassay kit. Serum HBV-DNA was quantified using a sensitive real-time polymerase chain reaction (RT-PCR) technique.

\section{Pentamers and PD1 analysis}

The following antibodies were used for PBMC staining: anti-CD8-FITC, anti-PD1-APC and anti-HLA-A2 (Bio 
Table 1 Patients demographic data and levels of serum markers at time of initial presentation

\begin{tabular}{|c|c|c|c|c|c|}
\hline Patient no. & Gender & $\begin{array}{l}\text { Level of serum } \\
\text { ALT ( IU/L) }\end{array}$ & $\begin{array}{l}\text { Level of serum } \\
\mathrm{HBsAg}(\mathrm{ng} / \mathrm{mL})\end{array}$ & $\begin{array}{l}\text { HBV-DNA } \\
\text { (copies/mL) }\end{array}$ & $\begin{array}{c}\text { Expression of } \\
\mathrm{HLA}-\mathrm{A}_{2}\end{array}$ \\
\hline 1 & male & 200 & 527 & $1 \times 10^{7}$ & positive \\
\hline 2 & male & 120 & 513 & $1.5 \times 10^{7}$ & positive \\
\hline 3 & male & 135 & 468 & $6.3 \times 10^{6}$ & positive \\
\hline 4 & female & 122 & 491 & $6.1 \times 10^{6}$ & positive \\
\hline 5 & male & 150 & 132 & $8.7 \times 10^{6}$ & positive \\
\hline 6 & female & 132 & 401 & $1.3 \times 10^{5}$ & positive \\
\hline 7 & female & 197 & 401 & $5.1 \times 10^{6}$ & positive \\
\hline 8 & male & 200 & 441 & $1.1 \times 10^{5}$ & positive \\
\hline 9 & male & 101 & 356 & $7.5 \times 10^{4}$ & positive \\
\hline 10 & female & 157 & 437 & $3.3 \times 10^{6}$ & positive \\
\hline 11 & male & 156 & 491 & $2.0 \times 10^{5}$ & positive \\
\hline 12 & female & 189 & 411 & $7.0 \times 10^{5}$ & positive \\
\hline 13 & female & 172 & 328 & $5.4 \times 10^{6}$ & positive \\
\hline 14 & male & 194 & 396 & $1.9 \times 10^{6}$ & positive \\
\hline 15 & female & 200 & 567 & $1.1 \times 10^{7}$ & positive \\
\hline
\end{tabular}

Abbreviations: $A L T$, alanine transaminase; $H B S A g$, hepatitis $B$ surface antigen; $H B V$, hepatitis $B$ virus; $H L A$, human leukocyte antigen.

Legend, San Diego, CA USA). HBV-specific CD8+ T-cells were identified with human leukocyte antigen A2 (HLA-A2) pentamers containing core 18-27 peptide (FLPSDFFPSV), evn 335-343 peptide (FLLTRILTI) and pol 575-583 peptide (FLLSLGIHL), which were labeled with the fluorochrome PE.

PBMCs from peripheral blood were incubated for $15 \mathrm{~min}$ at $37^{\circ} \mathrm{C}$ with $1 \mu \mathrm{g}$ of PE-labeled pentamer complex in PBS and then washed twice. Cells were then incubated with saturating concentrations of directly conjugated antiCD8-FITC and PD1-APC mAb, for 15 min. After washing the cells twice, they were analyzed immediately on a FACSCalibur flow cytometer using Cell Quest software (BD biosciences, East Rutherford NJUSA).

Lymphocytes were gated according to their physical parameters. All patients were HLA-A2 positive.

Pentamer-positive responses are reported as the percentage of pentamer-positive T-cells among the total CD8 population. Frequencies of pentamer-positive cells in healthy control were not exceeding $0.08 \%$ of total circulating CD8 cells. The expansion (n-fold) was calculated as the ratio of the percentage of pentamer-positive $\mathrm{T}$ cells between the $\mathrm{T} 1$ and $\mathrm{T} 2$ periods.

\section{B7H1 expression analysis}

B7-H1 expression on circulating myeloid dendritic cells (mDCs) was measured using $1 \mathrm{~mL}$ of fresh heparinized peripheral blood. Cells were lysed with FACS lysing solution (BD biosciences, East Rutherford NJUSA) to remove RBCs and then incubated with antibodies against B7-H1-PE and CD11c-PEcy7 for $20 \mathrm{~min}$ at room temperature. After washing twice with PBS, the cells were analyzed by flow cytometry on a FACSCalibur flow cytometer (BD Biosciences).

\section{Immunohistochemical staining}

Acetone-fixed liver tissue cryosections $(5 \mu \mathrm{m})$ were incubated with anti-PD-1, anti-PD-L1 and anti-CD8 (Abcam plc, Cambridge, UK) antibodies overnight at $4^{\circ} \mathrm{C}$ after blocking endogenous peroxidase activity with $0.3 \% \mathrm{H}_{2} \mathrm{O}_{2}$. Positive cells (brown color) were counted in high power fields (hpf) by 2 independent observers. PD-1 and PD-L1 immunofluorescence dual staining was performed. In brief, liver tissues were incubated overnight with primary antibodies at $4^{\circ} \mathrm{C}$ followed by secondary antibodies for 45 minutes at room temperature. Anti- PD-1 and PD-L1 (both diluted 1:50; Santa Cruz Biotechnology, Santa Cruz, CA USA) and anti- CD8, CD68, CD31, CD11c and (all diluted 1:50; Dako, Glostrup, Denmark) were used as primary antibodies. Fluorescein isothiocyanate- conjugated immunoglobulin $\mathrm{G}$ and fluorochrome phycoerythrin -conjugated immunoglobulin G (Santa Cruz Biotechnology) were used as secondary antibodies.

6. For RT-PCR, total RNA from liver biopsy tissue as indicated was isolated using RNeasy kits (Qiagen, Venlo, Netherlands). cDNA synthesis was performed using SuperScript One-Cycle cDNA kit (Life Technologies, Carlsbad, CA USA). The cDNA served as a template for real-time PCR using Fast SYBR Green Master kit (Life Technologies). The following primers for RT-PCR were used: human IFN $\gamma$ primers, 5' -GCATCCAAAAGAGT GTGGAG-3' (forward) and 5'-GCAGGCAGGACAACC 
ATTAC-3'(reverse); human TNF $\alpha$ primers 5'- GAGTGA CAAGCCTGTAGCC-3' (forward) and 5'-GAGGACCT GGGAGTAGATGA-3' (reverse); and human glyceralde hyde-3-phosphate dehydrogenase (GAPDH) primers, 5' -AACAGCGACACCCACTCCTC -3' (forward) and 5' -GGAGGGGAGATTCAGTGTGGT -3'(reverse). All reactions were performed in triplicate. IFN $\gamma$ and TNF $\alpha$ mRNA expression of different group specimens were normalized to glyceraldehydes 3-phosphate dehydrogenase (GAPDH). Relative mRNA levels are presented as unit values of $2^{-\Delta \Delta C t}$, where $C t$ is the threshold cycle value defined as the fractional cycle number at which the target fluorescent signal passes a fixed threshold above baseline.

\section{Statistical analysis}

The experimental data were analyzed using SPSS 13.0 software (IBM, Amonk, NY USA). Quantitative data are described using mean \pm standard deviation (SD). Comparisons between groups were analyzed using variance or nonparametric tests (Kruskal-Wallis and MannWhitney $\mathrm{U}$ tests). $\mathrm{p}<0.05$ was considered significant.

\section{Results}

\section{Clinical outcome}

At baseline, all enrolled patients had high serum viral and HBsAg loads. The highest HBV viral load was greater than $1.5 \times 10^{7}$ (copies $/ \mathrm{mL}$ ). The lowest HBV viral load was greater than $1 \times 10^{5}$ (copies $/ \mathrm{mL}$ ). The mean serum HBsAg load of 15 patients was $433.6 \pm 115.4(\mathrm{ng} / \mathrm{ml})$. During the Climax phase of inflammation (T2), a significant increase in serum ALT levels was paralleled by a profound decrease in the serum HBV load in all patients. Ten of 15 patients showed a low level of HBV-DNA replication from $5 \times 10^{3}$ to $1.5 \times 10^{4}$ copies $/ \mathrm{mL}$ by the $\mathrm{T} 4$ period while five patients had serum HBV-DNA less than $5 \times 10^{3}$ copies $/ \mathrm{mL}$.

\section{Longitudinal analysis of HBV-specific pentamer from T1 to $\mathrm{T} 4$}

Multiple HBV-specific $\mathrm{T}$ cell responses were detected throughout the clinical course from the $\mathrm{T} 1$ to the T4 period. During the T1 period, the levels of core 18-27, env335-343 and pol575-583 were $0.69 \% \pm 0.29 \%$, $0.84 \% \pm 0.33 \%$ and $0.94 \% \pm 0.30 \%$ respectively; significantly higher than the healthy control level (which never exceeded $0.08 \%$ ). During the T2 period, the frequency of core18-27, env335-343 and pol575-583 further increased significantly in all patients. Core $18-27$ was detectable at a frequency of $2.77 \% \pm 0.45 \%$ of total circulating CD8+ cells (1300 core18-27 cells in 50,000 CD8+ cells), reaching a frequency of $3.0 \%$ in 2 patients. The levels of env335-343 and pol575-583 in CD8+ cells also increased significantly, reaching the frequencies of $2.38 \% \pm 0.37 \%$ (1200 env 335-343 cells in 50,000 CD8+ cells) and
$2.93 \% \pm 0.54 \%$ ( 1460 pol $575-583$ cells in 50,000 CD8+ cells) respectively. During the decline phase of inflammation (T3), the frequency of core18-27, env335-343 and pol575-583 in total circulating CD8+ cells were decreased from $2.77 \% \pm 0.45 \%$ to $1.75 \% \pm 0.55 \%$, from $2.38 \% \pm 0.37 \%$ to $1.88 \% \pm 0.55 \%$ and from $2.93 \% \pm 0.54 \%$ to $1.99 \% \pm 0.16 \%$ respectively. During the regression phase of inflammation (T4), the frequency of these 3 pentamers further decreased to no more than $1 \%$ of total circulating CD8+ cells (Figure 1, Figure 2, Figure 3A).

\section{Longitudinal analysis of PD1 and PD-L1 expression from $\mathrm{T} 1$ to $\mathrm{T} 4$ in patients with chronic hepatitis $\mathrm{B}$}

The frequency of PD1 and PD-L1 expression on circulating CD8+ $\mathrm{T}$ cells and $\mathrm{mDCs}$ respectively were detected from the T1 to T4 periods. From T1 to T2, the proportion of PD1 positive cells increased significantly from $13.1 \% \pm 4.2 \%$ to $35.7 \% \pm 5.8 \%$ of total circulating CD8+ cells. During the T3 period, the proportion of PD1positive cells decreased significantly from $35.7 \% \pm 5.8 \%$ in the T2 period to $23.2 \% \pm 4.05 \%$ of total circulating CD8+ T cells. During the T4 period, the proportion of PD1-positive cells further decreased to $13.4 \% \pm 3.0 \%$, which approaches the levels found in the T1 period (Figure 4A, Figure 3C). The frequency of PD1 expression on pentamers was significantly higher than that among total circulating CD8+ T cells. During T2 period the frequency of PD1 expression on pentamers of core 18-27, env335-343 and pol575-583 were $57.5 \% \pm 13.6 \%$, $54.2 \% \pm 5.6 \%$ and $53.3 \% \pm 7.5 \%$ respectively (Figure $4 \mathrm{~B}$, Figure 5).

The dynamic PD-L1 expression was also detected on circulating $\mathrm{CD} 11 \mathrm{c}+\mathrm{mDCs}$ from the $\mathrm{T} 1$ to the $\mathrm{T} 4$ period. The level of PD-L1 expression on CD11c + cells increased significantly after the onset of liver inflammation. The highest frequencies of PD-L1and CD11c dual positive cells were found during the T2 period. Following $\mathrm{T} 2$, the frequency of PD-L1and CD11c dualpositive cells decreased progressively from $40.1 \% \pm 5.82 \%$ in T2 to $28.86 \pm 4.95 \%$ in T3 period. During T4, PD-L1 expression levels further decreased to11.2\% $\pm 3.54 \%$, which was lower than the levels detected in the $\mathrm{T} 1$ period (Figure 4C, Figure 3C).

\section{Longitudinal analysis of intrahepatic CD8, PD-1and PD-L1 expression from $\mathrm{T} 2$ to $\mathrm{T} 4$}

Serially sectioned liver biopsy specimens of enrolled patients from periods $\mathrm{T} 2$ to $\mathrm{T} 4$ period were examined for CD8, PD1 and PD-L1 expression by immunohistochemistry. During the T2 period, CD8+ positive cells infiltrated the portal tract and extend into the liver parenchyma. The number of infiltrating CD8+ positive cells decreased from $328 \pm 58 / \mathrm{hpf}$ in T2 to $186 \pm 21 / \mathrm{hpf}$ in T3 period. During regression phase of inflammation 


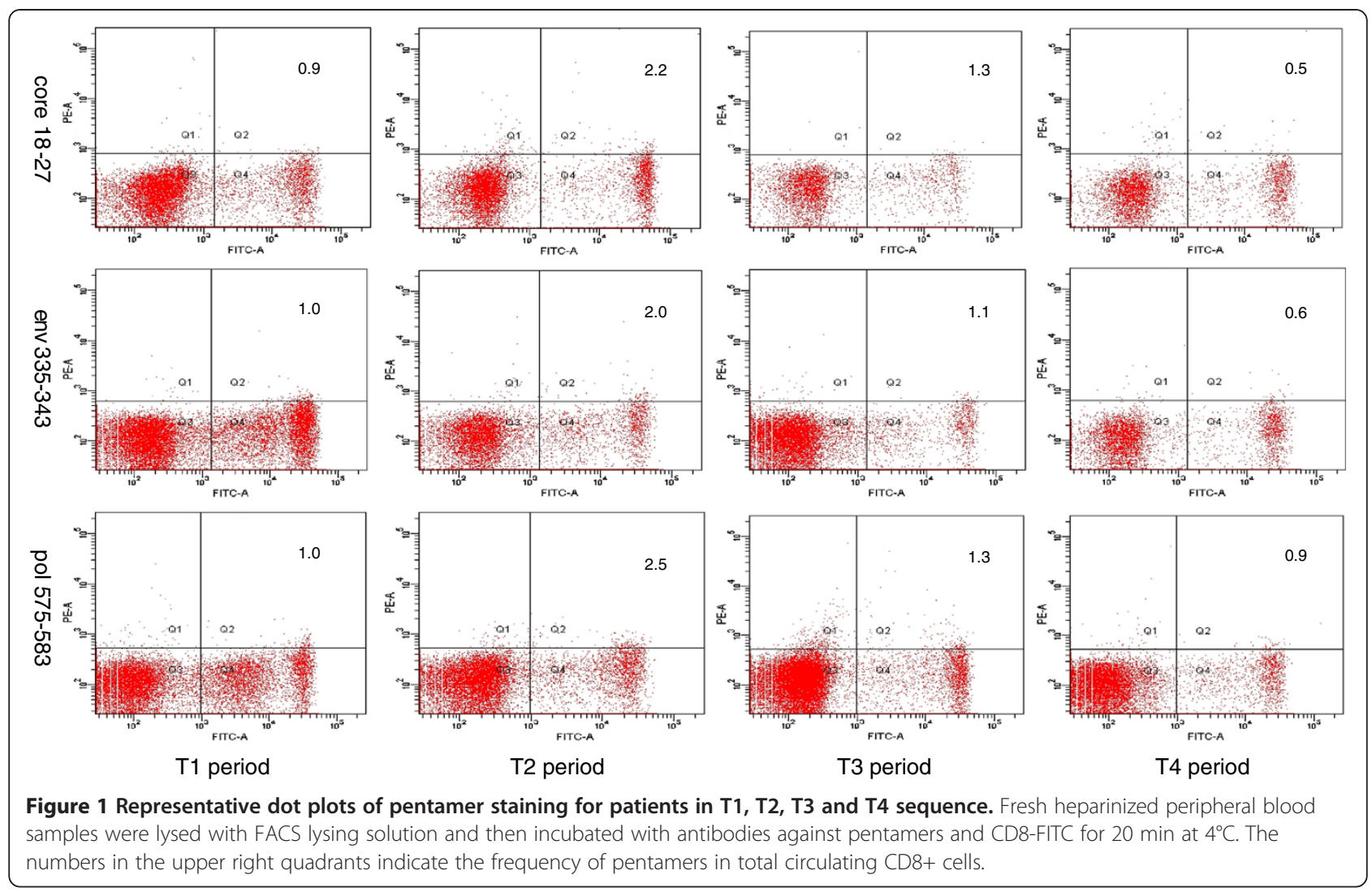

(T4), several CD8+ positive cells were observed in liver lobular area, most aggregated in fibrous septa (Figure 6, Figure 3D).

Using serial section, intra-hepatic PD1 and PD-L1 expression was also detected dynamically by immunohistology. During the T2 period, PD1 and PD-L1 positive cells were abundantly observed in periportal and lobular areas of the liver. During the T3 period, the numbers of PD1 and PD-L1 positive cells in the liver lobe area

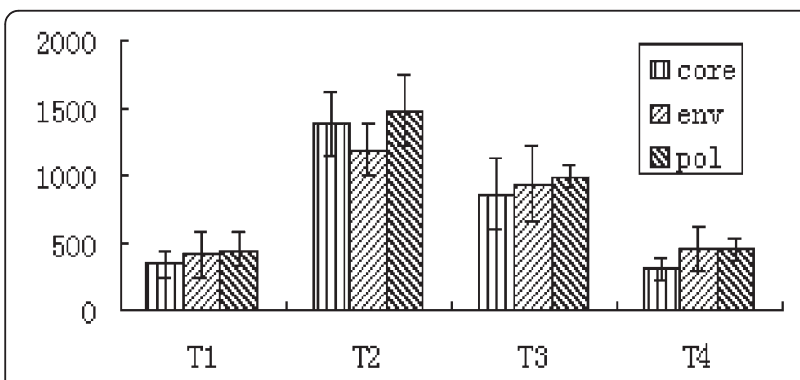

Figure 2 The numbers of core-, env- and pol-PE positive cells in $50000 \mathrm{CD} 8+$ cells were compared betweemT1,T2,T3 and T4. The number of core, env and pol increased significantly from $\mathrm{T} 1$ to $\mathrm{T} 2$, and decreased progressively from $\mathrm{T} 2$ to $\mathrm{T} 4$. core, env and pol, $n=15, P_{\text {T1 vs T2 }}<0.01, P_{\text {T2 vs T3 }}<0.05, P_{\text {T3 vs T4 }}<0.01$. decreased significantly. The mean number of infiltrating PD1 positive cells decreased from $388 \pm 122 /$ hpf in T2 to $174 \pm 53 / \mathrm{hpf}$ in T3. The number of infiltrating PD-L1-positive cells also decreased from $250 \pm 92 / \mathrm{hpf}$ in T2 to $103 \pm 14$ in T3. In T4, PD1 positive cells were almost absent in the lobular region, with most observed in fibrous septa and the portal tract, while PD-L1positive cells were also frequently observed in the lobular region of the liver. The number of PD-L1 positive cells decreased from $103 \pm 14 /$ hpf in T3 to $92 \pm 13$ /hpf in T4. There was no significant difference between the number of PD-L1-positive cells in T3 and T4 (Figure 6, Figure 3D).

\section{Co-localization of intra-hepatic PD-L1 with various cell markers in $\mathrm{T} 4$}

tlsb19ptThe number of intra-hepatic PD-L1 positive cells did not decrease significantly from T3 to T4 period. Immunofluorescence double staining of liver tissue from the T4 period showed that PD-L1 was primarily expressed on CD68-positive macrophages (Kupffer cells), and CD31-positive sinusoidal epithelial cells (Figure 7A). Intra-hepatic PD-L1 expression on CD11c + cells was also compared between T3 and T4 period. During T3, 

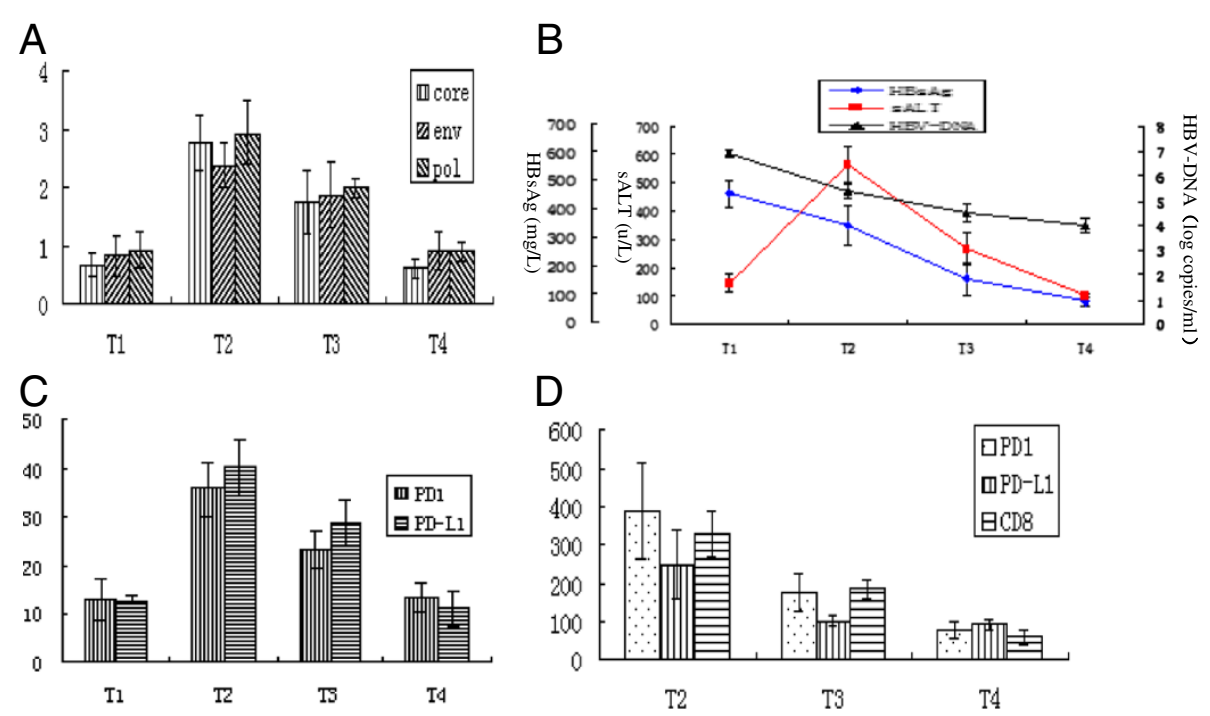

Figure 3 Correlation between PD1, PD-L1 expression and serum pentamer frequency, ALT level, HBsAg and HBV-DNA load.

(A) Percentage of core, even and pol from T1 to T4. The frequency of pentamers including core 18-27, even 335-343 and pol 575-583 increased upon initial of inflammation (T1), reached peak level during climax of inflammation (T2) and decreased gradually during regression of inflammation (T4). (core, even and pol, $\mathrm{n}=15, \mathrm{P}_{\mathrm{T} 1}$ vs $\mathrm{T} 2<0.01, \mathrm{P}_{\mathrm{T} 2}$ vs $\mathrm{T} 3<<0.05, \mathrm{P}_{\mathrm{T} 3}$ vs $\mathrm{T} 4<0.01$ ) (B) Fluctuation of $s A L T$, HBsAg and HBV-DNA from T1 to T4. From T1 to T2, serum ALT level increased significantly, in parallel with pentamer cells clonal expansion, and decreased significantly in concordance with pentamer cells clonal contraction from T3 to T4. ( SALT, $n=15, \mathrm{P}_{\mathrm{T} 1}$ vs T2 $<0.01, \mathrm{P}_{\mathrm{T} 2}$ vs T3 $<0.05, \mathrm{P}_{\mathrm{T} 3}$ vs T4 $<0.05$ ) Serum HBsAg and HBV-DNA load decreased during inflammation flare up period. (HBsAg and HBV-DNA copies, $n=15, P_{\text {T1 vs }} \mathrm{T}_{2}<0.01, \mathrm{P}_{\mathrm{T} 2}$ vs $\mathrm{T} 3<0.01$, $\mathrm{P}_{\text {T3 vs T4 }}<0.01$ ) (C) Percentage of circulating PD1 and PD-L1 from T1 to T4. From T1 to T2 period, in parallel with pentamer cells clonal expansion, circulating PD1 and PD-L1 expression increased significantly. From T3 to T4 period, parallel with pentamer cells clonal contraction,

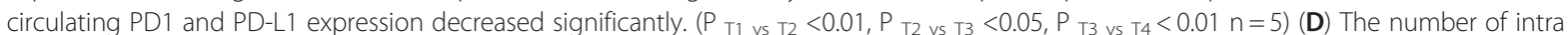
hepatic CD8, PD1 and PD-L1 positive cells from T2 to T4. DuringT2 period, the number of intra-hepatic PD1, PD-L1 and CD8 reached the highest level and they were decreased significantly in T3 period. From T3 to T4 period, number of intra-hepatic PD-1 and CD8 positive cells further decreased significantly, however PD-L1 did not. (PD1,PD-L1 and CD8, $P_{T 2}$ vs T3 $<0.01 ; P D 1$ and CD8, $P_{T 3}$ vs T4 $<0.01 ; P D-L 1, P$ T3 vs T4 $>0.05 ; n=5$ ).

most CD11c + cells expressed PD-L1 (Figure 7B); while during $\mathrm{T} 4$, the majority of $\mathrm{CD} 11 \mathrm{c}+$ cells did not (Figure 7C). Because the frequency of PD-L1 expression on circulating CD11c + cells decreased significantly from T3 to T4 period, there is good concordance between intra-hepatic and circulating PD-L1 expression on CD11c + cells.

\section{Dynamic analysis of TNF- $\alpha$ and IFN- $\gamma$ mRNA expression from $\mathrm{T} 1$ to $\mathrm{T} 4$}

Pro-inflammatory cytokines expression levels were also dynamically detected by real-time PCR in chronic hepatitis B patients from T1 to T4 period. As shown in Figure 8 , TNF- $\alpha$ and IFN- $\gamma$ mRNA can be detected in patients in T1 period, and increased progressively, reaching peak levels during the climax phase of inflammation (T2) and decreasing rapidly during the decline phase (T3). During T4, IFN- $\gamma$ mRNA levels decreased to less than those in the T1 period, while TNF- $\alpha$ mRNA expression were at a relatively high level. The TNF- $\alpha$ mRNA level further decreased from the $\mathrm{T} 3$ to the T4 period, but was still significantly higher than the levels found during T1 (Figure 8).

\section{Correlation between PD1/ PD- $\mathrm{L}_{1}$ expression and downstream inflammatory events}

As shown in Figure 3, the circulating and intra-hepatic PD1/PD1-L expression were correlated with downstream inflammatory events, such as HBV-specific CD8 T cells expansion and contraction, TNF $\alpha$ and IFN $\gamma$ mRNA expression and changes in serum HBV-DNA and ALT levels.

The number of PD1 and PD-L1 positive cells, both intra- and extra-hepatic increased significantly during $\mathrm{T} 2$ compared to those in $\mathrm{T} 1$ period. In particular intrahepatic PD1- and PD-L1-positive cells infiltrated extensively in the lobular and portal areas during T2. During the same phase, the number of pentamers such as core 18-27, pol 575-583 and even 335-343 also peaked rapidly, reaching levels greater than 4,3 and 3.2 times higher than the levels in T1 respectively. Subsequently, the serum ALT level also increased to more than 10 


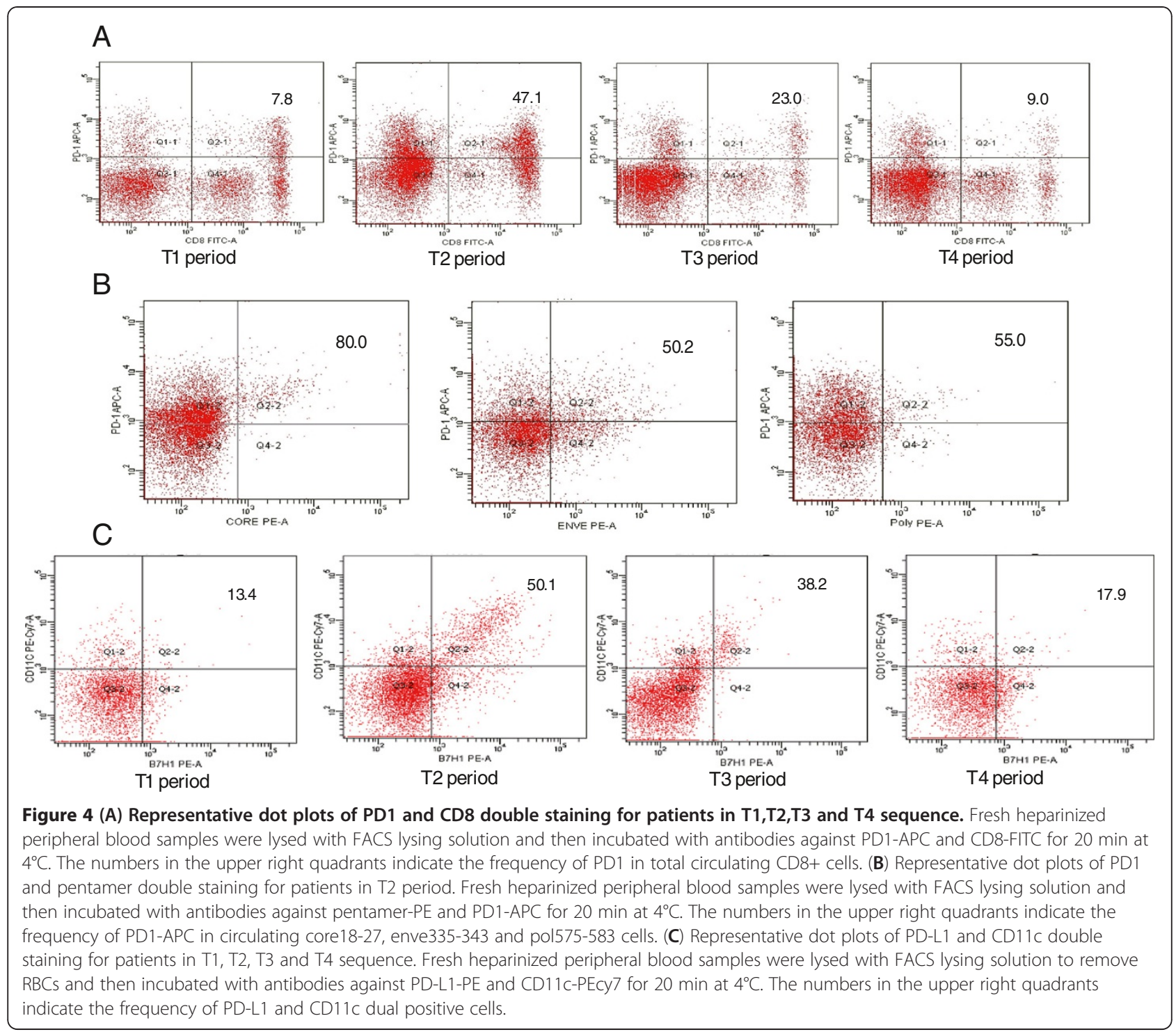

times greater than the upper limit of normal paralleling marked decrease in HBV-DNA and HBsAg. During this period, TNF $\alpha$ and IFNymRNA expression also reached peak levels.

During T3 period, the serum HBV-DNA levels progressively decreased to levels less than 10-fold below those found in T2, and serum HBsAg levels decreased greater than 2-fold. At the meantime, the number of pentamers, which include core18-27, even 335-343 and pol 575-583 cells also decreased significantly, paralleling the decrease of serum ALT, and intra-hepatic TNF $\alpha$ and IFNymRNA expression levels. Concomitant with these changes, the levels of both the circulating and intrahepatic PD1 and PD-L1 expression also decreased significantly.

During T4 period, serum HBV-DNA levels further decreased. The serum ALT also further declined, approaching normal level (less than 100u/L). Correlating very well with these changes, the frequency of circulating pentamers and the number of intra-hepatic CD8+ cells further decreased significantly and the intra-hepatic IFN- $\gamma$ mRNA expression level decreased to less than that found in T1; however, TNF $\alpha$ mRNA maintained a relatively high level throughout the same period. The frequency of circulating PD1-positive cells decreased near to T1 levels, and almost all of the liver infiltrating PD1-positive cells aggregate in fibrous septa. It is noted that during this period, the number of intra-hepatic PDL1-positive cells did not decrease significantly compared to that during the T3 period (Figure $3 \mathrm{~A}, \mathrm{~B}, \mathrm{C}, \mathrm{D}$ ).

\section{Discussion}

PD1, a negative regulator of the immune response, is predominantly expressed on activated $\mathrm{T}$ cells $[10,11]$. 


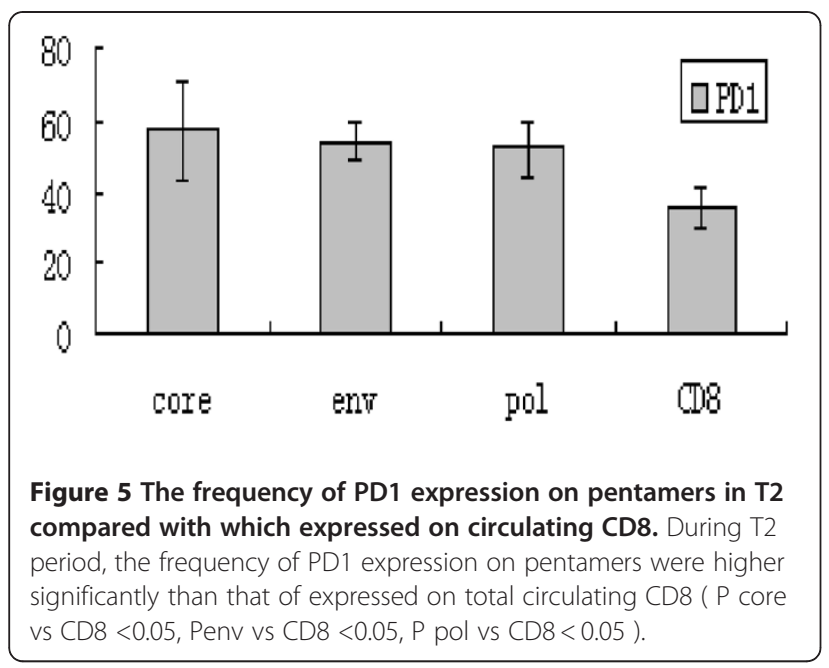

PD-L1, the ligand for PD1, is induced on APCs upon onset of activation $[10,11]$. The interaction between PD1 and PD-L1 transmits a co-inhibitory signal to downstream molecular events and then limits the activated $\mathrm{T}$ cell proliferation and cytotoxic effects, resulting in tissue protection against severe injury by an overvigorous immune response [12-14]. During the inflammation flare up period in patients with chronic hepatitis $\mathrm{B}$, the frequency of circulating PD1-positive cells and the number of intra-hepatic PD1-positive cells increased significantly and reached peak levels. During the same phase, circulating and intra-hepatic PD-L1 expression also increased significantly. Activated HBV specific $\mathrm{T}$ cells expressing PD1 were recruited to the liver and contacted PD-L1 expressing APCs (e.g. mDCs, kupffer cells, and LSECs). This interaction resulted in impaired functions of HBV specific-T cells. Kassel et al. reported that the upregulation of intrahepatic PD-L1 may efficiently limit over-vigorous immune response, avoiding severe liver damage [14]. Chen et al. also reported that delayed PD1 upregulation in patients with acute hepatitis B is closely associated with fulminant hepatitis[12].

Based on the Polly Matzinger's viewpoint, the expression of co-inhibitory molecules is needed for the maintenance of homeostasis [15]. In chronic hepatitis B patients, during the immune-inactive phase, whose liver function was nearly normal, it is not necessary to increase significantly for circulating and intra-hepatic PD1 and PD-L1 expression. Our previous studies revealed that in chronic hepatitis B patients with immuneinactive state, PD-L1 positive cells were almost absent in liver lobe (data is not shown). Rachel Kassel et al. also reported that PD1 and PD-L1 expression always increased significantly in inflamed liver from chronic

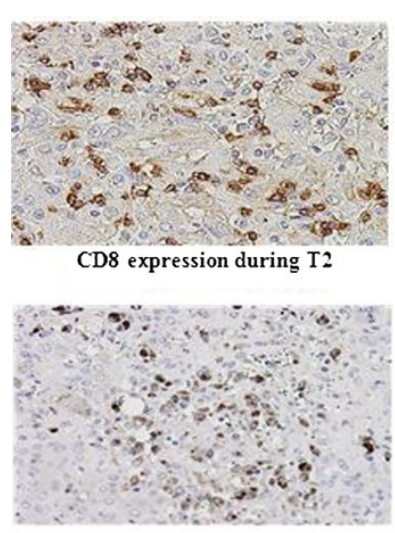

PDl expression during T2

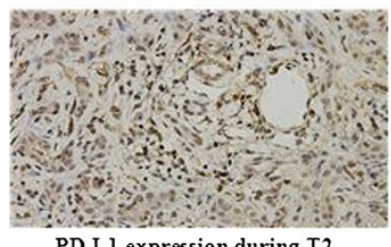

PD-L1 expression during T2

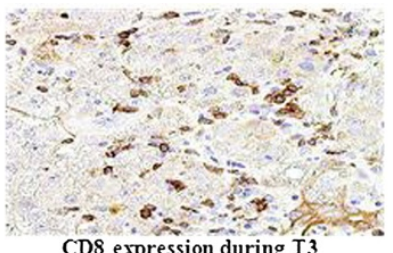

CD8 expression during T3

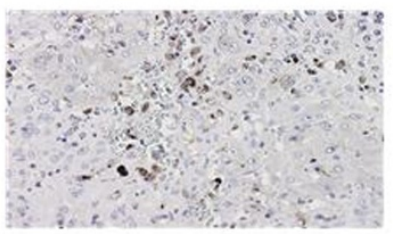

$\mathrm{PDl}$ expression during $\mathrm{T} 3$

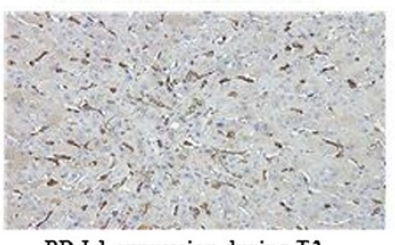

PD-Ll expression during T3

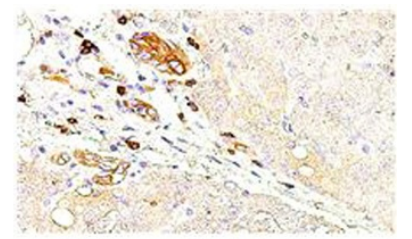

CD8 expression during T4

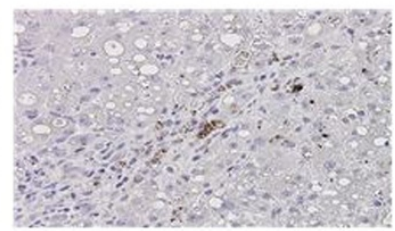

$\mathrm{PDl}$ expression during T4

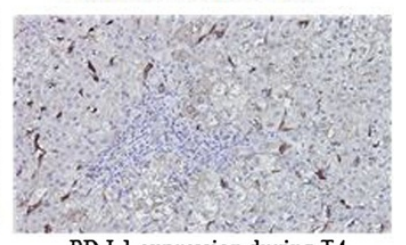

PD-Ll expression during T4

Figure 6 Immunohistochemical staining for intrahepatic CD8-positive, PD-1-positive and PD-L1-positive cells in patients with chronic hepatitis B. Representative figures for patients in T2, T3 and T4 sequence. $n=5$, High-power field, original magnification 400x. During T2 period, CD8-positive, PD-1-positive or PD-L1-positive cells infiltrated extensively in liver tissue and they were decreased significantly in T3. In T4, the CD8positive and PD-1-positive cells were less observed in lobular areas, while PD-L1 positive cells were also frequently observed in liver lobular region. 


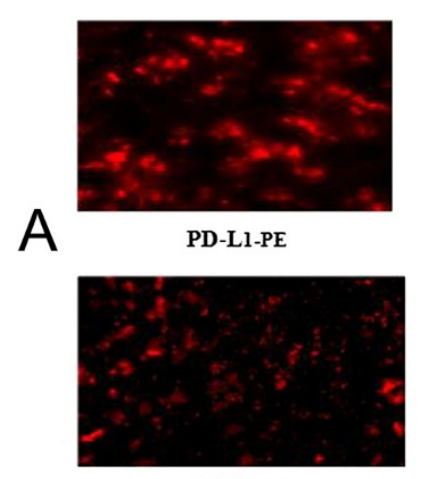

PD-Ll-PE

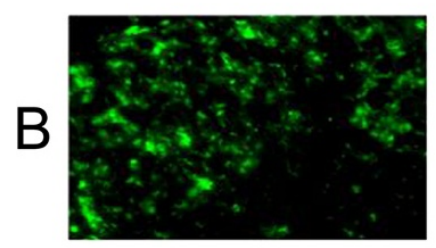

CDIlc-FITC

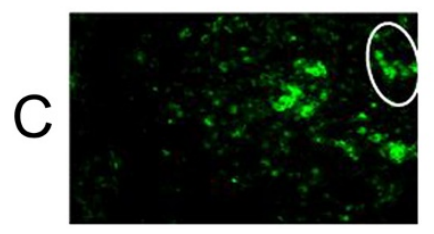

CDIlc-FITC

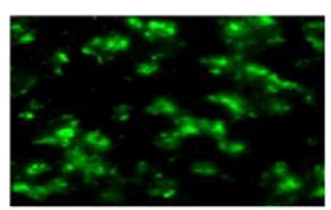

CD68-FITC

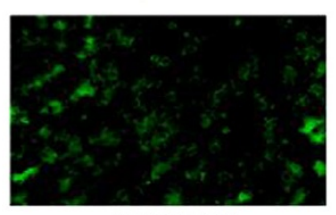

CD31-FITC

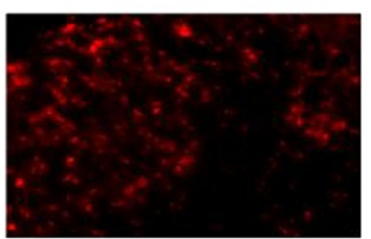

PD-L1-PE

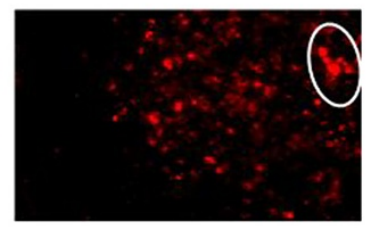

PD-L1-PE

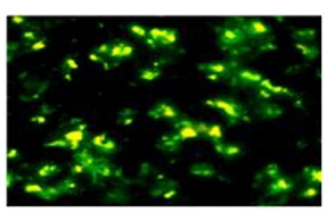

PD-L1/CD68

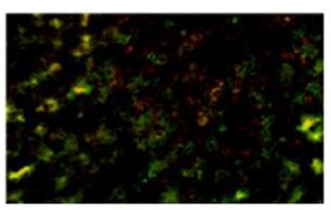

PD-L1/CD31

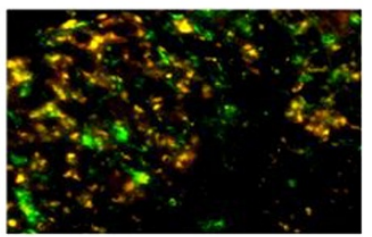

CDIlc/PD-Ll

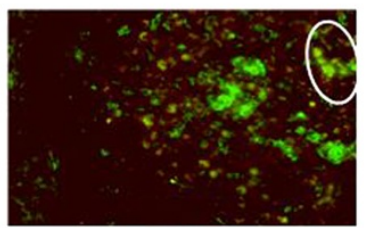

CDIlc/PD-Ll

Figure 7 (A) Co-localization of PD-L1 with CD31 and CD68 is shown by immunofluorescence double staining in liver biopsy specimens of patients with hepatitis B in T4. PD-L1 (red) is co-localized with CD31 (green) positive endothelia cells or CD68 (green) positive Kupffer cells. The 2-color merged panels show co-localization visible (yellow). Original magnification $\times 200$. (B) Co-localization of PD-L1 and CD11C in liver tissue from patients during T3. PD-L1 (red) is co-localized with CD11c (green). The 2-color merged panels show co-localization (yellow). Most

CD11c + cells expressed PD-L1. Original magnification $\times 200$. (C) Co-localization of PD-L1 and CD11c in liver tissue from patients in T4. PD-L1 (red) is co-localized with CD11c (green). The 2-color merged panels show co-localization (yellow). Most CD11c + cells did not express PD-L1. Only CD11c + cells in the circle expressed PD-L1. Original magnification $\times 200$.

hepatitis B patients with immune-clearance state [14].

Although the up-regulation of PD1 and PD-L1 may benefit restoration of liver function and maintenance of homeostasis, it is also thought to facilitate replication of remaining $\mathrm{HBV}$, finally resulting in persistent infection. In chronic hepatitis B patients, immune clearance phase and immune inactive phase always displayed alternatively. During the immune clearance phase, parallel with serum ALT elevation, serum HBV-DNA and HBsAg load declined, and may eventually lead to HBV-DNA seroclearance and HBsAg seroconversion to its antibody (anti-HBs) in part of patients; while following immune clearance phase, most of the patients enter an immune inactive phase with normal serum ALT, low serum HBV-DNA. In immune inactive phase, HBV-DNA may replicate actively again and HBV antigens may accumulated until it triggers a next round of immune response against HBV.
To elucidate the role of PD1 and PD-L1 in the mechanism of these two distinct states alternation, PD1 and PD-L1 expression were analyzed longitudinally in present study throughout the immune clearance phase; and the changes of PD1 and PD-L1 expression were related to HBV -specific T-cell clonal expansion, proinflammatory cytokine expression, serum ALT, HBV-DNA and HBs-Ag fluctuation.

During the T2 phase, HBV specific T cells, including core 18-27, even353-341 and pol 575-583, expanded rapidly reaching the levels more than $35,30,37$ times higher than healthy control level (which never exceeded $0.08 \%$ ), respectively. Concomitant with $\mathrm{HBV}$ - specific Tcell clonal expansion, serum ALT reached the peak levels, followed by a significant decreased in serum HBV-DNA and HBsAg load. During this period, the percentage of PD1 expression of total CD8 cells increased 


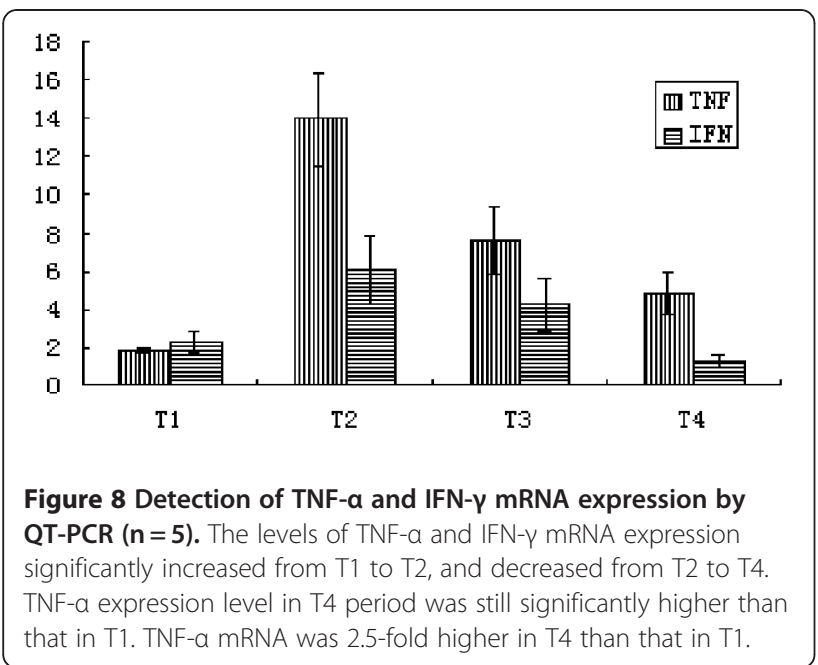

from $13.1 \% \pm 4.2 \%$ in $\mathrm{T} 1$ phase to $38.7 \% \pm 8.3 \%$ in $\mathrm{T} 2$ phase. The frequency of PD1 expression on pentamers was greater than $50 \%$ in the T2 phase.

During the T3 phase, the frequency of HBV-specific CD8 + T-cells decreased significantly, serum ALT level decreased by more than half of that in the T2 period, followed by further viremia reduction. During this period, the frequency of circulating PD1 expression further decreased significantly.

In T4, serum HBV-DNA further decreased to levels lower than those found in T3 and serum ALT level decreased to less than $100 \mathrm{IU} / \mathrm{L}$. The frequency of HBVspecific CD8 + T-cells further decreased to less than $1.0 \%$ and the frequency of circulating PD1 expression also decreased to less than the level found in T1.

Intra-hepatic PD1 expression was detected throughout the clinical course from $\mathrm{T} 2$ to $\mathrm{T} 4$. In the T2 phase, CD8+ and PD1+ cells infiltrated extensively in lobular and portal areas. The number of infiltrating PD1-positive cells decreased significantly from $388 \pm 122 / \mathrm{hpf}$ in T2 to $174 \pm 53 / \mathrm{hpf}$ in T3. In the T4, the number of intrahepatic PD1+ cells further decreased significantly, and most PD1-positive cells could be observed aggregated in fibrous septa and in periportal regions. Our previous findings showed that almost all liver-infiltrating CD8 T-cells expressed the home receptor CCR5 and CCR7 (data is not shown). This phenomenon was consistent with previous results that PD1-positive CD8 T-cells home from peripheral circulation to lobular and portal areas to form portal tract- associated lymphoid tissue. [16-18].

Two conclusions can be drawn based on these data. First, CD8 T cells maintain normal proliferative capacity towards HBV antigens and undergo colon expansion during inflammatory episodes in patients with chronic hepatitis B. Second, PD1 expression is located primarily on activated HBV specific $\mathrm{T}$ cells and changes are associated with HBV-specific T-cell clonal expansion and contraction, serum ALT and viral load changes. These data are in line with previous findings which showed that PD1 expression is induced and upregulated significantly on activated HBV-specific CD8 Tcells during colon expansion period $[8,12,14]$.

PD-L1 expression exhibits a similar dynamic pattern in patients with chronic hepatitis $\mathrm{B}$. The expression of PD-L1 on circulating CD11c $+\mathrm{mDCs}$ increased during the initial phase of liver inflammation (T1) and reached peak levels in parallel with up-regulation of the frequency of PD1 expression on circulating CD8+ T cells in the T2 period. In the T3 period, following a decline in circulating PD1 expression, the frequency of PD-L1 expression also decreased significantly. In the regression phase (T4), associated with the decline of PD1 expression, the frequency of circulating PD-L1 decreased further, approaching to the levels in T1 period. These data indicate that there is good concordance between circulating PD-1 and PD-L1; it changes during the inflammation flare period.

Intra-hepatic PD-L1 expression was detected from T2 to T4 phases of chronic hepatitis B. During the climax phase of inflammation (T2), intra-hepatic PD-L1 positive cells were observed extensively in lobular areas. The number of these cells decreased significantly in the decline phase of inflammation (T3) compared to the levels in T2. It did not decrease significantly from T3 to T4 period although the frequency of circulating PD-L1 decreased significantly from $28.86 \% \pm 4.95 \%$ to $11.18 \% \pm 3.54 \%$ in the same period. Note, in T3 period, most of liver infiltrating CD11c + cells were PD-L1 positive, while in T4, intrahepatic PD-L1 positive cells were CD68+ Kupffer and CD31+ endothelial cells, and the majority of intrahepatic CD11c were PD-L1 negative.

Studies have reported that PD-L1 expression can be induced by pro-inflammatory cytokines such as IFNyand TNF $\alpha$ in an antigen-independent manner [19-22]. During the regression period (T4), the mRNA expression level of TNFa was maintained at a higher level compared to that during the $\mathrm{T} 1$ period. This result suggests that in liver tissue, a relatively high level of TNF $\alpha$ expression may attribute to intrahepatic PD-L1 up-regulation in the T4 phase.

In conclusion, these results suggest that persistent upregulation of intra-hepatic PD-L1 on CD68 kupffer and CD31 endothelia may constitute an immunosuppressive microenvironment during the regression phase (T4). The effects of PD1 expressing HBV-specific T-cells are dampened when they home the liver and come in contact with PD-L1-expressing CD68+ Kupffer and CD31+ endothelial cells. This may facilitate replication of remaining HBV-DNA and finally lead to recurrence of hepatitis. 


\section{Conclusion}

In general, the levels of co-inhibitory molecules expression were increasing progressively in parallel with ascending inflammation and were decreasing significantly during inflammatory regression phase. Contrary to this phenomenon, intra-hepatic PD-L1 expression on non-parenchyma cells did not decrease significantly in inflammatory regression phase. This may contribute to constitute immunosuppressive microenvironment which may damp HBV-specific immune response and finally lead to persistent HBV infection.

\section{Competing interest}

The authors declare that they have no competing interests.

\section{Authors' contributions}

Zhang Wenjin contributed to study concept, study design, writing of the manuscript. Zheng Shusen contributed to study concept and study design. Peng Chuanhui contributed to data acquisition, statistical analysis. Wan Yunle contributed to data acquisition, statistical analysis. Shaikh Abdul Lateef contributed to writing of the manuscript. All authors read and approved the final manuscript.

Support by: Zhejiang Provincial Natural Science Foundation, No. Y2110169.

\section{Author details}

'Key Laboratory of Combined Multi-organ Transplantation, Ministry of Public Health, First Affiliated Hospital, School of Medicine, Zhejiang University, 79 Qingchun Road, Hangzhou 310003, China. 'Department of Hepatobiliary Surgery, Sun Yat-Sen Memorial Hospital, Sun Yat-Sen University, Guangzhou 510120, Guangdong Province, China.

Received: 11 March 2012 Accepted: 8 August 2012

Published: 16 August 2012

\section{References}

1. Peng G, Li S, Wu W, et al: PD-1 upregulation is associated with HBVspecific T cell dysfunction in chronic hepatitis B patients. Mol Immunol 2008, 45:963-970.

2. Evans A, Riva A, Cooksley H, et al: Programmed Death 1 Expression During Antiviral Treatment of Chronic Hepatitis B: Impact of Hepatitis B eAntigen Seroconversion. Hepatology 2008, 48:759-769.

3. Rutebemberwa A, Ray SC, Astemborski J, et al: High-Programmed Death-1 Levels on Hepatitis C Virus-Specific T Cells during Acute Infection Are Associated with Viral Persistence and Require Preservation of Cognate Antigen during Chronic Infection1. J Immunol 2008, 181:8215-8225.

4. Urbani S, Amadei B, Tola D, et al: PD-1 Expression in Acute Hepatitis C Virus (HCV) Infection Is Associated with HCV-Specific CD8 Exhaustion. J Virol 2006, 80:11398-11403.

5. Jeong H-Y, Lee $Y-J$, Seo S-K, et al: Blocking of monocyte-associated B7-H1 (CD274) enhances HCV-specific T cell immunity in chronic hepatitis $C$ infection. J Leukoc Biol 2008, 83:755-764.

6. Carolina B, Paola F, Caterina V: Characterization of Hepatitis B Virus (HBV)Specific T-Cell Dysfunction in Chronic HBV Infection. J Virol 2007, 81:4215-4225.

7. Victoria K, Wiesch JSz, Thomas K, et al: High Level of PD-1 Expression on Hepatitis C Virus (HCV)-Specifi CD8+ and CD4+ T Cells during Acute HCV Infection, Irrespective of Clinical Outcome. J Virol 2008, 82:3154-3160.

8. Lucy G-M, Brent P, Jared K, et al: Upregulation of PD-1 Expression on Circulating and Intrahepatic Hepatitis C Virus-Specific CD8+ T Cells Associated with Reversible Immune Dysfunction. J Virol 2007, 81:9249-9258.

9. Selenko-Gebauer N, Majdic O, Szekeres A, et al: B7-H1 (Programmed Death-1 Ligand) on Dendritic Cells Is Involved in the Induction and Maintenance of T Cell Anergy. J Immunol 2003, 170:3637-3644.

10. Iwai $Y$, Terawaki S, Ikegawa M, et al: PD-1 Inhibits Antiviral Immunity at the Effector Phase in the Liver. J. Exp. Med 2003, 198:39-50.
11. Karrar A, Broome U, Uzunel M, et al: Human liver sinusoidal endothelial cells induce apoptosis in activated T cells: a role in tolerance induction. Gut 2007, 56:243-252.

12. Chen $\mathrm{L}$, Zhang $\mathrm{Z}$, Chen $\mathrm{W}$, et al: B7-H1 up-regulation on myeloid dendritic cells significantly suppresses $T$ cell immune function in patients with chronic hepatitis B. J Immunol 2007, 178:6634-6641.

13. Yu MC, Chen CH, Liang X, et al: Inhibition of T-cell responses by hepatic stellate cells via B7-H1-mediated T-cell apoptosis in mice. Hepatology 2005, 40:1312-1321.

14. Kassel R, Cruise MW, lezzoni JC, et al: Chronically inflamed livers upregulate expression of inhibitory $B 7$ family members. Hepatology 2009 50(5):1625-1637.

15. Poly M: Tolerance, danger, and the extended family. Annu. Rev.Immunol 1994, 12:991-1045.

16. By Hiroyuki Y, Kenjiro M, Yanyun Z, et al: Regulation by chemokines of circulating dendritic cell precursors, and the formation of portal tractassociated lymphoid tissue, in a granulomatous liver disease. J. Exp. Med 2001, 193:35-49.

17. Hiroyuki $Y$, Kenjiro M, Yanyun Z, et al: Regulation by chemokines of circulating dendritic cell precursors, and the formation of portal tractassociated lymphoid tissue, in a granulomatous liver disease. J. Exp. Med 2001, 193:35-49.

18. Grant AJ, Goddard S, Ahmed-Choudhury J, et al: Hepatic expression of secondary lymphoid chemokine (CCL21) promotes the development of portal-associated lymphoid tissue in chronic inflammatory liver disease. Am J Pathol 2002, 160:1445-1455.

19. Mazanet MM, Hughes $\mathrm{CC}$, et al: $\mathrm{B} 7-\mathrm{H} 1$ is expressed by human endothelial cells and suppresses T cell cytokine synthesis. J Immunol 2002, 169:3581-3588.

20. Rodig N, Ryan T, Allen J: A., Greenfield, E A, et al.: Endothelial expression of PD-L1 and PD-L2 down-regulates CD8+ T cell activation and cytolysis. Eur J Immunol 2003, 33:3117-3126.

21. Muhlbauer M, M Fleck C, Schutz T, et al: PD-L1 is induced in hepatocytes by viral infection and by interferon- $a$ andyand mediates $T$ cell apoptosis. J Hepatol 2006, 45:520-528.

22. Abhishek D, Matthew $H$, Nathan $D$, et al: Functional skewing of the global CD8 T cell population in chronic hepatitis B virus infection. J Exp Med 2008, 205(9):2111-2124.

doi:10.1186/1471-230X-12-109

Cite this article as: Wenjin et al:: Longitudinal fluctuations in PD1 and PD-L1 expression in association with changes in anti-viral immune response in chronic hepatitis B. BMC Gastroenterology 2012 12:109.

\section{Submit your next manuscript to BioMed Central and take full advantage of:}

- Convenient online submission

- Thorough peer review

- No space constraints or color figure charges

- Immediate publication on acceptance

- Inclusion in PubMed, CAS, Scopus and Google Scholar

- Research which is freely available for redistribution 